\title{
Absolute Configuration of Solenopsis Piperidines is a Tool to Classify Fire Ants (Formicidae:Myrmicinae)
}

\author{
Francisca D. S. Araújo, ${ }^{a}$ Marcela A. Botelho, ${ }^{a}$ José R. Trigo ${ }^{b}$ and Anita J. Marsaioli*,a \\ ${ }^{a}$ Instituto de Química and ${ }^{b}$ Departamento de Biologia Animal, Instituto de Biologia, \\ Universidade Estadual de Campinas, PO Box 6154, 13084-971 Campinas-SP, Brazil
}

\begin{abstract}
Piperidine alkaloids are predominant in ant venom glands of $S$. invicta and $S$. saevissima. These ants are broadly sympatric in Southeastern Brazil, and potentially diagnostic compounds could assist in ant identification. We determined the absolute configuration of 2,6-dialkyl-piperidines of S. invicta and S. saevissima workers and used these data to group Southeastern Brazil species. The monitoring of venom samples by chiral gas chromatography coupled to a flame ionization detector (GC-FID) revealed that $S$. saevissima produces the four stereoisomers of 2-methyl-6-undecylpiperidines, 2-methyl-6-tridecyl-piperidines and 2-methyl-6-tridecenyl-piperidines, while $S$. invicta venom consisted primarily of a single enantiomer cis and trans from each piperidine alkaloid. Our findings showed that the absolute configuration of piperidine alkaloids of fire ant venom may be a potential chemical tool to distinguish $S$. invicta and $S$. saevissima sympatric species in Southeastern Brazil.
\end{abstract}

Keywords: Solenopsis saevissima, Solenopsis invicta, piperidine alkaloid, absolute configuration, chemotaxonomy

\section{Introduction}

Ants of the genus Solenopsis (Hymenoptera: Formicidae), known as fire ants, produce a variety of oxygen-free polyketide alkaloids in their venom glands such as piperidine, piperideine, pyrrolidine, indolizidine, and pyrrolizidine alkaloids. Piperidine alkaloids predominate in the constitution of most Solenopsis species and can be classified as solenopsins, isosolenopsins, dehydrosolenopsins, or dehydroisosolenopsins..$^{1-3}$

The most widely studied Solenopsis species are S. invicta, S. richteri, S. geminata, S. saevissima, S. xyloni, and $S$. aurea. ${ }^{3}$ The significant morphological and behavioral similarity of some species has prompted studies to assist in their identification, which is hindered by the existence of intermediate hybrids. ${ }^{5}$ An important tool to overcome this problem is the analysis of potentially diagnostic compounds, such as 2,6-dialkyl-piperidines and cuticular hydrocarbons. ${ }^{6,7}$

Dall' Aglio-Holvorcem et al. ${ }^{6}$ analyzed two chemical classes in widely separated populations of S. invicta and S. saevissima. They found that piperidine alkaloids clustered S. invicta, but not $S$. saevissima, while cuticular hydrocarbons strongly clustered both species. However, this study did not take into account the absolute configuration of alkaloids. Our laboratory has developed a rapid methodology for efficient determination of the absolute configuration of 2-methyl-6-alkylpiperidine using chiral gas chromatography coupled to a flame ionization detector (GC-FID) ${ }^{4}$ Comparison of the chromatographic profiles of synthetic racemic mixtures of 2-methyl-6-undecylpiperidines with venom samples of $S$. saevissima workers and queens from different nests showed the natural occurrence of four stereoisomers of 2-methyl-6-undecylpiperidine in both trifluoroacetylated and acetylated samples. These results demonstrated that trans alkaloids are not always $(2 R, 6 R)$ and cis alkaloids are not always $(2 R, 6 S)$, contrasting with previously reported data. ${ }^{2}$

In order to find chemical criteria to separate $S$. saevissima and $S$. invicta within an area of $75,000 \mathrm{~km}^{2}$ in Southeastern Brazil, the present study aimed to determine the absolute configuration of 2,6-dialkyl-piperidines and use these data to group the Southeastern Brazil species.

*e-mail: anita@iqm.unicamp.br 


\section{Experimental}

Ants

The eighteen fire ant alkaloid samples were the same as those used by Dall' Aglio-Holvorcem et al. ${ }^{6}$ These samples were stored at $-20{ }^{\circ} \mathrm{C}$ for five years and the alkaloid pattern had not changed. Thirty larger worker ants of $S$. invicta and $S$. saevissima species were collected in three individual nests at six localities in São Paulo State, in Southeastern Brazil. ${ }^{6}$ The locality codes for $S$. invicta samples were Instituto Agronômico de Assis, Assis (AS); Companhia Agrícola Brejo das Almas, Botucatu (BT); Fazenda Experimental/ UNIMAR, Marília (MA); Bosque-Zoológico Municipal, São José do Rio Preto (RP); and for S. saevissima: Parque Pedra do Baú, Campos do Jordão (CJ); Parque Estadual Intervales, Capão Bonito, (INT). To extract the alkaloids, the large worker ants were manually homogenized for $10 \mathrm{~min}$ in a test tube containing sea-sand (Fluka, Geneva, Switzerland) and $2 \mathrm{~mL}$ of dichloromethane (residual pesticide grade, Carlo Erba, Cer Brasil, SP, Brazil). ${ }^{6}$

\section{Chemicals}

All solvents were treated with anhydrous sodium sulfate (Synth, SP, Brazil) and distilled or bidistilled water. Pyridine and ethyl ether were treated with calcium hydride and distilled. Column chromatography purifications were carried out with silica gel (Acros Organics, Acros, SP, Brazil, 0.035-0.070 mm) impregnated with silver nitrate (Merck, SP, Brazil). Trifluoroacetic anhydride (SigmaAldrich, SP, Brazil), copper sulfate (Vetec, Sigma-Aldrich, SP, Brazil), acetyl chloride (Sigma, SP, Brazil), potassium carbonate (Merck, SP, Brazil), iodine (Rothyl, SP, Brazil), dimethyl disulfide (Acros Organics, SP, Brazil), sodium thiosulfate (Vetec, SP, Brazil), anhydrous magnesium sulfate (Nuclear, SP, Brazil), 10\% Pd/C (Aldrich, SP, Brazil), and sodium bicarbonate (Merck, SP, Brazil) were purchased.

Instrumentation

Gas chromatography coupled with mass spectrometry (GC-MS) analyses $(70 \mathrm{eV})$ were carried out with an Agilent 6890 chromatograph coupled to a Hewlett-Packard 5973 mass detector, equipped with a DB5 fused silica capillary column $(30 \mathrm{~m} \times 0.25 \mathrm{~mm} \times 0.25 \mu \mathrm{m}$ i.d.). Mass spectra were recorded over the 40-600 amu range at 3.54 scans s$^{-1}$ from $\mathrm{m} / \mathrm{z} 40$ to 600 . Helium was the carrier gas at a flow rate of $1 \mathrm{~mL} \mathrm{~min}^{-1}$. The injector and interface temperatures were kept at 250 and $280^{\circ} \mathrm{C}$, respectively. The oven temperature program was $50-290^{\circ} \mathrm{C}$ at $25^{\circ} \mathrm{C} \mathrm{min}^{-1}$, and then maintained for $20 \mathrm{~min}$ at $290{ }^{\circ} \mathrm{C}$. One-microliter samples $\left(1 \mathrm{mg} \mathrm{mL}^{-1}\right)$ were injected using a 10:1 split ratio.

GC-FID analyses were performed using an Agilent 6890 chromatograph equipped with a Chrompack Chirasil CB chiral column $(25 \mathrm{~m} \times 0.25 \mathrm{~mm} \times 0.25 \mu \mathrm{m})$. High-purity hydrogen was the carrier gas at a flow rate of $2 \mathrm{~mL} \mathrm{~min}^{-1}$. $1 \mu \mathrm{L}$ samples were injected, with a 1:100 split ratio. The temperature program was $130-180{ }^{\circ} \mathrm{C}\left(0.3{ }^{\circ} \mathrm{C} \mathrm{min}^{-1}\right)$, maintained for $30 \mathrm{~min}$ at $180^{\circ} \mathrm{C}$, with the detector at $250{ }^{\circ} \mathrm{C}$ and the injector at $220^{\circ} \mathrm{C}$.

\section{Trifluoroacetylation reaction}

Approximately $1 \mathrm{mg}$ of ant venom samples containing alkaloids were solubilized in dry ethyl ether ( $1 \mathrm{~mL})$. Pyridine $(0.8 \mathrm{~mL})$, previously treated with calcium hydride, was added and trifluoroacetic anhydride $(100 \mu \mathrm{L})$ was slowly added to the reaction mixture, which was maintained in a glycerin bath overnight at $30^{\circ} \mathrm{C}$ with magnetic stirring. The reaction was finalized by adding ethyl acetate $(2 \mathrm{~mL})$ and a saturated aqueous solution of copper sulfate (the procedure was repeated three times). The organic phase was removed, dried with anhydrous sodium sulfate, concentrated in nitrogen flow and analyzed by GC-MS and chiral GC-FID. ${ }^{4}$

\section{2-Methyl-6-undecylpiperidine trifluoroacetamide}

GC-MS (electrospray ionization (EI) $70 \mathrm{eV}$ ), $\mathrm{m} / \mathrm{z}$ (\%): $349\left(\mathrm{M}^{+}, 0.1\right), 334$ (1), 280 (3), 222 (1), 209 (1), 194 (100), 178 (1), 152 (2), 140 (5), 126 (1), 95 (1), 81 (8), 69 (3), 55 (12), 41 (4).

\section{2-Methyl-6-tridecenylpiperidine trifluoroacetamide}

GC-MS (EI $70 \mathrm{eV}), \mathrm{m} / \mathrm{z}(\%): 375\left(\mathrm{M}^{+\cdot}, 2\right), 360$ (16), 306 (100), 281 (3), 267 (1), 253 (1), 234 (2), 220 (9), 194 (94), 178 (2), 166 (4), 152 (5), 140 (12), 126 (3), 109 (3), 96 (7), 81 (25), 67 (14), 55 (34), 41 (16).

\section{2-Methyl-6-tridecylpiperidine trifluoroacetamide}

GC-MS (EI $70 \mathrm{eV}), \mathrm{m} / \mathrm{z}(\%): 377\left(\mathrm{M}^{+}, 0.1\right), 362$ (1), 308 (2), 281 (1), 194 (100), 180 (1), 166 (1), 152 (1), 140 (4), 126 (1), 95 (1), 81 (7), 69 (4), 55 (11), 41 (5).

\section{2-Methyl-6-pentadecenylpiperidine trifluoroacetamide}

GC-MS (EI $70 \mathrm{eV}), \mathrm{m} / \mathrm{z}$ (\%): $403\left(\mathrm{M}^{+}, 1\right), 388$ (4), 334 (30), 262 (1), 234 (1), 220 (2), 194 (100), 180 (2), 166 (1), 152 (2), 140 (6), 121 (1), 109 (2), 95 (3), 81 (12), 67 (6), 55 (20), 41 (8).

2-Methyl-6-pentadecylpiperidine trifluoroacetamide GC-MS (EI $70 \mathrm{eV}), \mathrm{m} / \mathrm{z}$ (\%): 405 (M+., 0.1), 390 (1), 
$336(3), 280$ (2), 252 (1), 194 (100), $180(1), 152(1), 139$

(3), 126 (1), 113 (1), 96 (2), 81 (7), 69 (4), 55 (12), 43 (9).

\section{Catalytic hydrogenation microderivatization reaction}

Approximately 0.2 to $0.5 \mathrm{mg}$ of ant venom containing a mixture of trifluoroacetylated alkaloids, ethyl acetate $(1 \mathrm{~mL})$, and catalytic amount of $\mathrm{Pd} / \mathrm{C}(10 \%)$ was added to a round-bottom flask $(5 \mathrm{~mL})$. The flask was sealed with a rubber septum, connected to a hydrogen reservoir (1 atm), and magnetically stirred for $6 \mathrm{~h}$. The reaction was filtered using a silica gel column ( $1 \mathrm{~g}, 1 \mathrm{~cm}$ diameter), eluted with ethyl acetate $(10 \mathrm{~mL})$ and analyzed by GC-MS and GC-FID (chiral column). ${ }^{8}$

Purification of unsaturated ant venom alkaloids by silver nitrate-silica gel chromatography

Silica gel impregnated with silver nitrate was prepared as previously described. ${ }^{9}$ Thus, a Pasteur pipette $(1 \mathrm{~cm}$ diameter) was wrapped in aluminum foil and packed with silver nitrate/ silica gel in hexane. The sample was then added in the form of pastilles to silica gel. Elution was performed using a hexane/ ethyl ether gradient (100:0, 90:1 and 80:20, v/v). Fraction composition was determined by GC-MS, demonstrating a 90:1 gradient elution for saturated trifluoroacetylated alkaloids and an 80:20 (v/v) gradient elution for unsaturated alkaloids. Fractions containing saturated trifluoroacetylated alkaloids were analyzed by GC-FID, while those containing unsaturated trifluoroacetylated alkaloids were hydrogenated for GC-FID analysis.

\section{Derivatization reaction with DMDS/ $\mathrm{I}_{2}$}

Trifluoroacetylated samples of fire ant venom containing approximately $1 \mathrm{mg}$ were solubilized in dichlromethane $(50 \mu \mathrm{L})$ and added with dimethyl disulfide (DMDS, $500 \mu \mathrm{L}$ ) and iodine solution ( $40 \mu \mathrm{L}$ of $60 \mathrm{mg}$ of $\mathrm{I}_{2}$ in $1 \mathrm{~mL}$ distilled ether). The reaction was stirred overnight at $40^{\circ} \mathrm{C}$ under refluxing. The reactions were stopped with $2 \mathrm{~mL}$ of aqueous sodium thiosulfate $\left(\mathrm{Na}_{2} \mathrm{~S}_{2} \mathrm{O}_{3}, 1 \mathrm{~g}\right.$ in $10 \mathrm{~mL}$ of distilled water). The organic phase was extracted, dried with anhydrous magnesium sulfate, filtered through treated cotton and evaporated under flowing nitrogen. The derivatized samples were dissolved in bidistilled hexane and analyzed by GC-MS. ${ }^{10}$

DMDS derivatives of 2-methyl-6-tridecenylpiperidine trifluoroacetamides

GC-MS (EI $70 \mathrm{eV}), m / z(\%): 469\left(\mathrm{M}^{+}, 9\right), 422(1), 406$ (1), 374 (6), 360 (2), 324 (1), 296 (100), 280 (2), 248 (10),
226 (6), 194 (31), 173 (57), 152 (25), 135 (16), 109 (6), 87

(22), 69 (24), 61 (24), 55 (40), 41 (16).

DMDS derivatives of 2-methyl-6-pentadecenylpiperidine trifluoroacetamides

GC-MS (EI $70 \mathrm{eV}), \mathrm{m} / z$ (\%): $497\left(\mathrm{M}^{+}, 10\right), 324$ (98), 276 (11), 253 (2), 194 (100), 173 (42), 140 (13), 95 (14), 81 (42), 55 (46), 41 (18).

Chemometric analysis

Cluster analysis, using Euclidean distance measures and minimum variance algorithm, was performed with the first diastereomeric $(\mathrm{dr})$ or enantiomeric ratio (er) of piperidine alkaloid trifluoroacetamides as variables and the individual nest at each site as sample, totalizing seven variables and eighteen samples (three individual nests in six localities). It was used only seven variables [C11:0 dr cis:trans, $\mathrm{C} 11: 0$ er cis $(2 R, 6 S: 2 S, 6 R), \mathrm{C} 11: 0$ er trans $(2 R, 6 R: 2 R, 6 S)$, C13:0 er cis en1:en2, C13:0 er trans en1:en2, C13:1 dr cis:trans, C13:1 er trans en1:en2], since the other eight were not present in all samples (see Table 1).

\section{Results and Discussion}

We developed a method to separate diatereomeric and enantiomeric piperidine alkaloids in the natural venoms in two species of fire ants (S. invicta and S. saevissima) trying to use these alkaloids as a chemotaxonimic tool to separate both species. We used large worker of three nests from six localities in São Paulo state to extract the alkaloids. After the trifluoroacetylation reaction (Figure S1 in the Supplementary Information (SI) section) and GC-FID analyses using the modified method proposed by Pianaro et al., ${ }^{4}$ natural venom containing piperidine alkaloids showed overlapping peaks in the chromatogram region where unsaturated alkaloids were eluted (Figure 1a). The peaks corresponding to the 2-methyl-6-tridecenylpiperidine stereoisomers were broad with partially overlapping peaks of 2-methyl-6-tridecylpiperidine stereoisomers, preventing good chiral discrimination and the possibility of co-injection with enantiomerically-enriched synthetic products. Thus, saturated and unsaturated alkaloids were separated using silver nitrate-silica gel chromatography (Figure S2 in the SI section). The fraction containing saturated alkaloids was directly analyzed by chiral GC-FID, and the fraction containing the unsaturated trifluoroacetylated alkaloids was submitted to catalytic hydrogenation (Scheme S1 in the SI section) to obtain the corresponding saturated derivatives. As such, compounds containing unsaturation in the side chain were converted 
Table 1. Monitoring of diastereomeric (dr) and enantiomeric (er) ratios of piperidine alkaloid trifluoroacetamides in Solenopsis venom samples

\begin{tabular}{|c|c|c|c|c|c|c|c|c|c|c|c|c|c|c|c|}
\hline $\begin{array}{l}\text { Nest } \\
\text { in each } \\
\text { locality }\end{array}$ & $\begin{array}{c}\mathrm{dr} \\
\text { cis:trans } \\
\text { C11:0 }\end{array}$ & $\begin{array}{c}\text { er } c i s \\
(2 R, 6 S: 2 S, 6 R) \\
\text { C11:0 }\end{array}$ & $\begin{array}{c}\text { er trans } \\
(2 R, 6 R: 2 R, 6 S) \\
\text { C11:0 }\end{array}$ & $\begin{array}{c}\mathrm{dr} \\
\text { cis:trans } \\
\text { C13:0 }\end{array}$ & $\begin{array}{c}\text { er } c i s \\
\text { en1:en2 } \\
\text { C13:0 }\end{array}$ & $\begin{array}{c}\text { er trans } \\
\text { en1:en2 } \\
\text { C13:0 }\end{array}$ & $\begin{array}{c}\mathrm{dr} \\
\text { cis:trans } \\
\text { C13:1 }\end{array}$ & $\begin{array}{c}\text { er } c i s \\
\text { en1:en2 } \\
\text { C13:1 }\end{array}$ & $\begin{array}{c}\text { er trans } \\
\text { en1:en2 } \\
\text { C13:1 }\end{array}$ & $\begin{array}{c}\mathrm{dr} \\
\text { cis:trans } \\
\text { C15:0 }\end{array}$ & $\begin{array}{c}\text { er } c i s \\
\text { en1:en2 } \\
\text { C15:0 }\end{array}$ & $\begin{array}{c}\text { er trans } \\
\text { en1:en2 } \\
\text { C15:0 }\end{array}$ & $\begin{array}{c}\mathrm{dr} \\
\text { cis:trans } \\
\text { C15:1 }\end{array}$ & $\begin{array}{c}\text { er } c i s \\
\text { en1:en2 } \\
\text { C15:1 }\end{array}$ & $\begin{array}{c}\text { er trans } \\
\text { en1:en2 } \\
\text { C15:1 }\end{array}$ \\
\hline AS01 & $31: 69$ & $100: 0$ & 100:0 & 1:99 & 100:0 & $95: 5$ & 1:99 & 100:0 & 100:0 & 0:100 & - & 100:0 & $0: 100$ & - & 100:0 \\
\hline AS02 & 49:51 & $100: 0$ & 100:0 & $10: 90$ & 100:0 & $100: 0$ & 9:91 & $100: 0$ & $100: 0$ & $0: 100$ & - & 100:0 & $0: 100$ & - & 100:0 \\
\hline AS05 & $34: 76$ & 100:0 & 100:0 & $5: 95$ & 100:0 & $97: 2$ & $3: 94$ & 100:0 & 100:0 & $0: 100$ & - & 100:0 & $0: 100$ & - & 100:0 \\
\hline BT01 & $12: 88$ & 100:0 & 100:0 & 1:99 & $100: 0$ & 100:0 & 2:98 & 100:0 & 100:0 & $0: 100$ & - & $100: 0$ & 1:99 & 100:0 & 100:0 \\
\hline BT02 & $14: 86$ & 100:0 & 100:0 & $5: 95$ & $100: 0$ & $100: 0$ & 3:97 & 100:0 & $100: 0$ & $0: 100$ & - & $100: 0$ & $60: 40$ & $0: 100$ & 100:0 \\
\hline BT03 & 43:57 & $100: 0$ & 100:0 & $28: 72$ & 100:0 & 100:0 & $37: 63$ & 100:0 & 100:0 & 0:100 & - & 100:0 & 23:77 & 100:0 & 100:0 \\
\hline MA01 & $35: 65$ & 100:0 & 100:0 & $0: 100$ & 0 & $98: 2$ & 1:99 & 100:0 & 100:0 & 0:100 & - & 100:0 & $0: 100$ & - & 100:0 \\
\hline MA02 & $56: 44$ & 100:0 & 100:0 & $0: 100$ & 0 & 100:0 & $16: 84$ & 100:0 & 100:0 & $0: 100$ & - & 100:0 & $0: 100$ & - & 100:0 \\
\hline MA05 & $39: 61$ & 100:0 & 100:0 & $0: 100$ & 0 & 100:0 & 2:98 & $53: 47$ & $100: 0$ & $0: 100$ & - & 100:0 & $0: 100$ & - & 100:0 \\
\hline RP03 & $62: 38$ & $100: 0$ & 100:0 & $0: 100$ & 0 & $84: 16$ & $0: 100$ & 0 & $100: 0$ & - & - & - & - & - & - \\
\hline RP04 & $46: 54$ & $100: 0$ & 100:0 & $0: 100$ & 0 & $80: 20$ & $0: 100$ & 0 & 100:0 & - & - & - & - & - & - \\
\hline RP05 & $37: 63$ & 100:0 & 100:0 & $0: 100$ & 0 & $96: 4$ & $0: 100$ & 0 & 100:0 & - & - & - & - & - & - \\
\hline CJ01 & $41: 59$ & $85: 15$ & $20: 80$ & $45: 55$ & $76: 24$ & $47: 53$ & $54: 46$ & 90:10 & $53: 47$ & $17: 83$ & 100:0 & 100:0 & $*$ & $*$ & * \\
\hline CJ02 & $48: 52$ & $84: 16$ & $77: 23$ & $28: 72$ & $46: 54$ & $94: 6$ & $24: 76$ & $51: 49$ & $96: 4$ & $33: 67$ & 100:0 & 100:0 & $*$ & * & $*$ \\
\hline CJ05 & $52: 48$ & $77: 23$ & $37: 63$ & $46: 54$ & $81: 19$ & $46: 54$ & $59: 41$ & $85: 15$ & $44: 56$ & $47: 53$ & $65: 35$ & $55: 45$ & $*$ & $*$ & $*$ \\
\hline INT2 & $52: 48$ & $66: 33$ & $37: 63$ & $53: 47$ & $71: 29$ & $56: 44$ & $62: 37$ & $72: 28$ & $66: 34$ & - & - & - & * & * & * \\
\hline INT3 & 43:57 & $53: 47$ & $77: 23$ & $42: 58$ & $57: 43$ & $69: 31$ & $32: 68$ & $53: 47$ & $93: 7$ & - & - & - & $*$ & $*$ & $*$ \\
\hline INT5 & $29: 71$ & $90: 10$ & $93: 7$ & $32: 68$ & $43: 57$ & $82: 18$ & $34: 66$ & $83: 17$ & $92: 8$ & - & - & - & $*$ & $*$ & * \\
\hline $\begin{array}{l}\text { Synthetic } \\
\text { standard }\end{array}$ & $79: 21$ & $48: 52$ & 49:51 & $97: 3$ & $49: 51$ & $42: 58$ & $97: 3$ & 49:51 & $42: 58$ & 99:1 & $47: 53$ & $51: 49$ & 99:1 & $47: 53$ & $51: 49$ \\
\hline
\end{tabular}

aThe abbreviations AS (Assis), BT (Botucatu), MA (Marília), RP (São José do Rio Preto), CJ (Campos do Jordão), INT (Intervalos, Capão Bonito) represent the localities where the nest was sampled. The number after abbreviations represent the number of nest assigned in by Dall'Aglio-Holvorcem et al. ${ }^{6}$ en $1: e n 2:$ enantiomer1:enantiomer2; -: compound not found; *: overlapping peaks.

into their saturated products without any chemical modification in the central chiral portion of the piperidine ring. Catalytic hydrogenation was extremely efficient, converting reactants to products almost quantitatively (Figure S3, in the SI section). After semi-purification and hydrogenation of unsaturated trifluoroacetylated alkaloids, chiral GC-FID analysis was fast and simple, exhibiting less overlapping (Figure 1b) and enabling coinjection with enantiomerically-enriched synthetic standards. Analysis of 2-methyl-6-tridecenylpiperidine (Figure S4 in the SI section) and 2-methyl-6-pentadecenylpiperidine (Figure S5 in the SI section) trifluoroacetamide DMDS derivatives revealed a double bond at carbon 4 and 6 of the alkyl chains, respectively.

Monitoring of enantiomeric and diastereomeric ratios of 2-methyl-6-undecylpiperidine trifluoroacetamides of Solenopsis venom samples from seven different localities in São Paulo State, Southeastern Brazil, showed

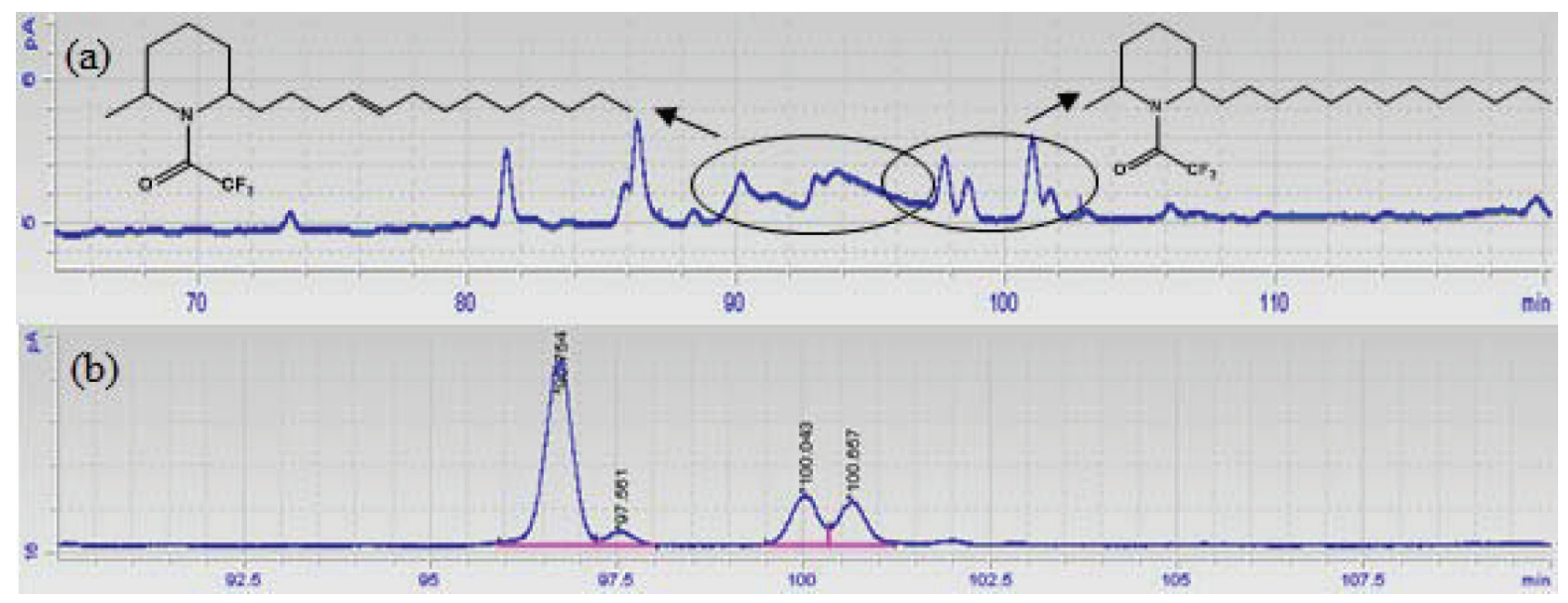

Figure 1. (a) Chiral GC-FID chromatogram of trifluoroacetylated sample of piperidine alkaloids of Solenopsis saevissima from Intervales, Capão Bonito, with the (b) expanded region of 2-methyl-6-tridecylpiperidine after semi-purification. 
significant changes in the diastereomeric ratio for all seven groups (Table 1). Identification of each stereoisomer of $( \pm)$-cis and $( \pm)$-trans-2-methyl-6-undecylpiperidine trifluoroacetamide was previously determined ${ }^{4}$ using queen venom samples with high enantiomeric ratios of each cis and trans isomer, which, after purification, provided $(2 R, 6 S)$-2-methyl-6-undecylpiperidine and $(2 R, 6 R)$-2-methyl-6-undecylpiperidine, respectively. Our results corroborated those reported by Pianaro et al.,$^{4}$ and the four stereoisomers of 2-methyl-6-undecylpiperidine trifluoroacetamides were present in the samples collected in localities CJ and INT (Table 1). These two groups of samples corresponded to the $S$. saevissima nests. The S. invicta nests showed the presence of a single enantiomer of cis- and trans-2-methyl-6-undecylpiperidine trifluoroacetamides.

The absolute configurations of 2-methyl6-tridecylpiperidine, 2-methyl-6-tridecenylpiperidine, 2-methyl-6-pentadecylpiperidine, and 2-methyl6-pentadecenylpiperidine (Table 1) trifluoroacetamides were expressed as enantiomer1:enantiomer2 (en1:en2) ratios because the absolute configuration of these piperidines was not determined. Variability was also observed in the diastereomeric ratios of these alkaloids for all seven sample groups. The four stereoisomers of cis- and trans-2-methyl6-tridecylpiperidine and cis- and trans-2-methyl6-tridecenylpiperidine trifluoroacetamides were also present in the S. saevissima samples. However, 2-methyl- 6-pentadecylpiperidine was not present in INT samples and showed variability in $\mathrm{CJ}$ samples, while the absolute configuration of 2-methyl-6-pentadecenylpiperidine was not determined due to peak overlays.

The cluster analysis was performed using three nests at six different localities and seven diastereomeric or enantiomeric ratio of piperidine alkaloid trifluoroacetamides (see Experimental section for more details). The analysis showed that is possible to group the two species (S. saevissima and S. invicta) using the absolute configuration of the stereogenic center of 2-methyl6-undecylpiperidine, 2-methyl-6-tridecylpiperidine and 2-methyl-6-tridecenylpiperidine (Figure 2). The nests of $S$. saevissima (localities CJ and INT) were separated from S. invicta (localities AS, BT and MA). However, S. invicta from RP locality clustered near $S$. saevissima. It is interesting to note that the RP population of S. invicta showed different alkaloid and hydrocarbon profiles from other S. invicta populations, suggesting an undescribed species. ${ }^{6}$ Due to the small nest sample in each locality (only 3), it is difficult to explain why some S. invicta populations (AS and BT) clustered together, while others did not (MA and RP). Geographical variation, hybrids, cryptic species or even unicoloniality ${ }^{11}$ have been hypothesized, but further studies with a large number of nests and molecular genetic analysis are needed to clarify this issue.

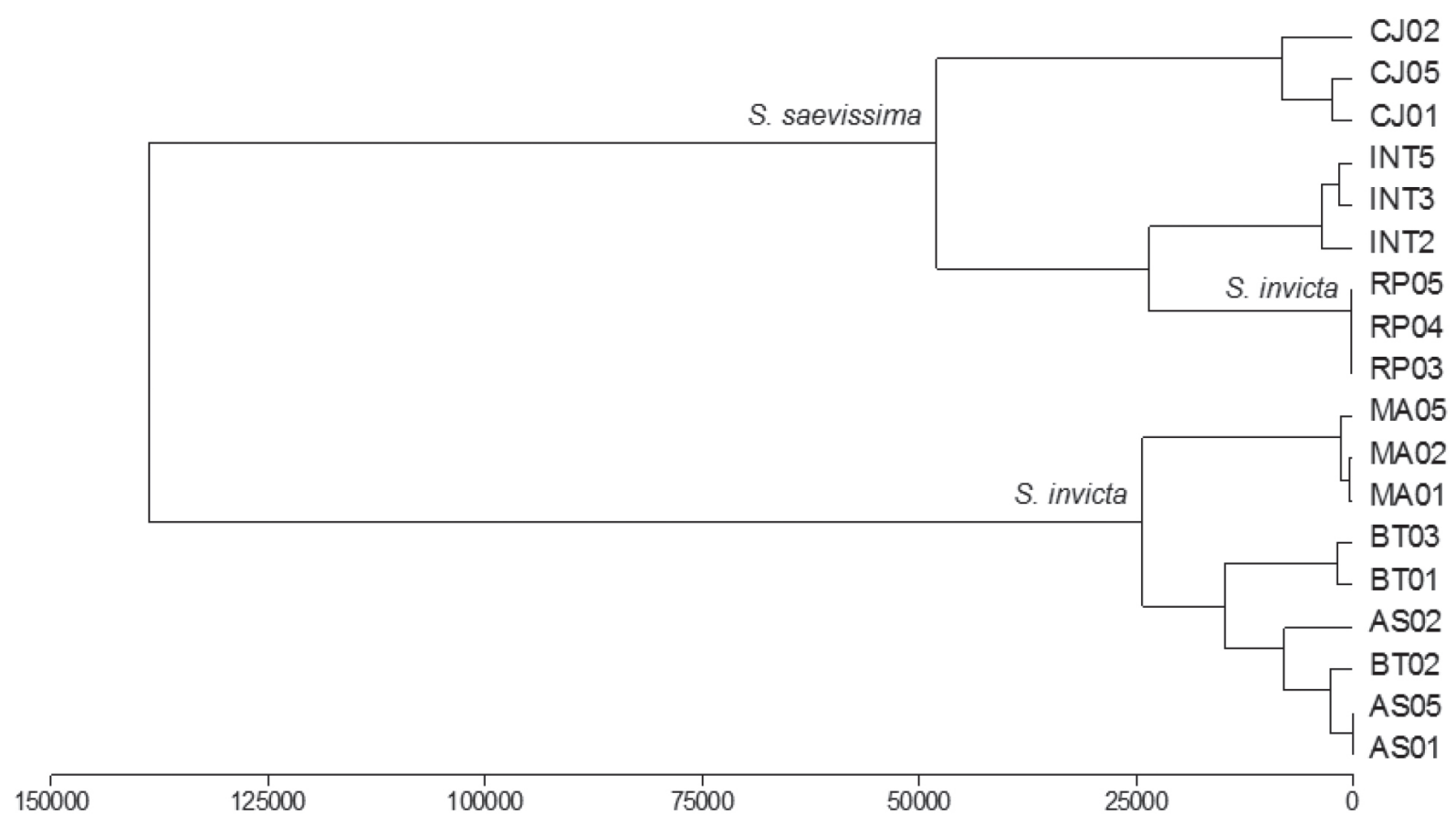

Squared Euclidean Distance

Figure 2. Clustering analysis of piperidine alkaloid stereiosomers of Solenopsis venom samples from Southeastern Brazil. See footnotes in Table 1 for abreviations. 


\section{Conclusions}

S. invicta and S. saevissima venom analysis of seven different regions of São Paulo State, Brazil, revealed the presence of four stereoisomers of 2-methyl-6-undecylpiperidines, 2-methyl-6-tridecyl-piperidines and 2-methyl6-tridecenyl-piperidines in S. saevissima, and the predominance of a single enantiomer cis and trans in each 2,6-dialkyl-piperidine in S. invicta. Our findings showed the potential of the absolute configuration of the piperidine alkaloid of fire ant venom as a chemical tool to distinguish the sympatric species $S$. invicta and $S$. saevissima in Southeastern Brazil. By integrating a natural synthetic product approach with classical taxonomic identification, we were able to improve the accuracy of Solenopsis identification. An integrative prespective combining, for example, molecular genetics with natural products, ${ }^{12}$ may contribute to solving taxonomic problems in several organisms.

\section{Supplementary Information}

Supplementary information is available free of charge at http://jbcs.sbq.org.br as PDF file.

\section{Acknowledgments}

The authors thank FAPESP (São Paulo Research Foundation) (grant 2012/18670-0) for the financial support.

\section{References}

1. Chen, J.; Cantrell, C. L.; Shang, H. W.; Rojas, M. G.; J. Agric. Food Chem. 2009, 57, 3128; Chen, L.; Fadamiro, H. Y.; Toxicon 2009, 53, 469; Chen, L.; Fadamiro, H. Y.; Toxicon 2009, 53, 479; Blum, M. S.; Fales, H. M.; Leadbetter, G.; Leonhardt,
B. A.; Duffield, R. M. A.; J. Nat. Toxins 1992, 1, 57; Jones, T. H.; Blum, M. S.; Fales, H. M.; Tetrahedron 1982, 38, 1949; MacConnell, J. G.; Blum, M. S.; Fales, H. M.; Tetrahedron 1971, 27, 1129; Pedder, D. J.; Fales, H. M.; Jaouni, T.; Blum, M.; MacConnell, J.; Crewe, R. M.; Tetrahedron 1976, 32, 2275; Brand, J. M.; Blum, M. S.; Fales, H. M.; MacConnell, J. G.; Toxicon 1972, 10, 259

2. Leclercq, S.; Thirionet, I.; Broeders, F.; Daloze, D.; Vander Meer, R. K.; Braekman, J. C.; Tetrahedron 1994, 50, 8465.

3. Attygalle, A. B.; Morgan, E. D.; Chem. Soc. Rev. 1984, 13, 245.

4. Pianaro, A.; Fox, E. G. P.; Bueno, O. C.; Marsaioli, A. J.; Tetrahedron: Asymmetry 2012, 23, 635.

5. Trager, J. C.; J. N. Y. Entomol. Soc. 1991, 99, 141; Tschinkel, W. R.; The Fire Ants, The Belknap Press of Harvard University Press: Cambridge, MA, 2006; Vander Meer, R. K.; Lofgren, C. S.; Fla. Entomol. 1988, 71, 323; Vander Meer, R. K.; Lofgren, C. S.; Alvares, F. M.; Fla. Entomol. 1985, 68, 501.

6. Dall'Aglio-Holvorcem, C. G.; Benson, W. W.; Gilbert, L. E.; Trager, J. C.; Trigo, J. R.; Biochem. Syst. Ecol. 2009, 37, 442.

7. Porter, S. D.; Vander Meer, R. K.; Pesquero, M. A.; Campiolo, S.; Fowler, H. G.; Ann. Entomol. Soc. Am. 1995, 88, 570.

8. Pomini, A. M.; Cruz, P. L. R.; Gai, C.; Araújo, W. L.; Marsaioli, A. J.; J. Nat. Prod. 2009, 72, 2125.

9. Andreão, P. S. S.; Giacomini, R. A.; Stumbo, A. M.; Waldman, W. R.; Braz-Filho, R.; Ligiéro, C. B. P.; Miranda, P. C. M. L.; Quim. Nova 2010, 33, 212.

10. Buser, H.-R.; Arn, H.; Guerin, P.; Rauscher, S.; Anal. Chem. 1983, 55, 818 .

11. Lenoir, A.; Devers, S.; Touchard, A.; Dejean, A.; Insect Sci. 2015, 23, 739.

12. Touchard, A.; Dauvois, M.; Arguel, M.-J.; Petitclerc, F.; Leblanc, M.; Dejean, A.; Orivel, J.; Nicholson, G. M.; Escoubas, P. J.; Proteomics 2014, 105, 217.

Submitted: April 12, 2017 Published online: August 24, 2017 